\title{
Interaction between Chondroitin-6-Sulfate and Entamoeba histolytica as Revealed by Force Spectroscopy
}

${ }^{1}$ Evander de J.O. Batista, ${ }^{1}$ Wanderley de Souza, ${ }^{2,3}$ Gilza M.P. Prazeres, ${ }^{3}$ Paulo M. Bisch and ${ }^{3}$ Gilberto Weissmüller

${ }^{1}$ Laboratório de Ultraestrutura Celular Hertha Meyer - Instituto de Biofísica Carlos Chagas Filho - Universidade Federal do Rio de Janeiro - RJ - Brasil. e-mail:ebatista@biof.ufrj.br ${ }^{2,3}$ Departamento de Química- Universidade Federal do Maranhão - MA - Brasil, ${ }^{3}$ Laboratório de Física Biológica - Instituto de Biofísica Carlos Chagas Filho - Universidade Federal do Rio de Janeiro - RJ - Brasil.

Entamoeba histolytica is a protozoan parasite that causes amebic dysentery in humans. Infection with this parasite may result in invasion of intestine. During this process, Entamoeba is able to kill and ingest epithelial cells, cells from the immune system and red blood cells. The cytoadhesion process exerted by the pathogen is regulated by chemical surface molecules. Essential components for the successful ingestion of cells are surface receptors of Entamoeba that mediate rapid recognition and uptake. Entamoeba histolytica has a $10 \mathrm{~nm}$ thick plasma membrane, with an external and continuous coat mainly composed by carbohydrate residues of glycoprotein. Only few surface molecules of Entamoeba have been described, as the $270 \mathrm{kDa}$ galactose $/ \mathrm{N}$-acetyl galactosamine (Gal/GalNAc) and $220 \mathrm{kDa} \mathrm{N}$ acetyl glucosamine-binding lectins, and the $112 \mathrm{kDa}$ surface adhesin[1]. The Gal/GalNAc lectin has been shown to mediate adherence of the parasite to exposed terminal galactose $/ \mathrm{N}$ acetyl-galactosamine residues of target cell glycoproteins or colonic mucins [2]. Trophozoites poorly adhere to target cells defective in production of $\mathrm{N}$ - and O-linked Gal-terminal oligosaccharides, thus confirming this carbohydrate specificity [3]. The Gal/GalNAc lectin is a heterodimer molecule composed of a heavy $(170 \mathrm{kDa})$ transmembrane subunit and a light $(31 / 35 \mathrm{kDa})$ glycosylphosphatidylinositol-anchored subunit linked by disulfide bonds [4].

Some interaction properties of Gal/GalNAc lectin and its ligands have been characterized using isolated E. histolytica membranes. For instance, Adler et al., (1995) showed that the interaction of GalNAc with membrane fractions is stronger when carried out in solutions with high ionic concentrations (above $50 \mathrm{mM} \mathrm{NaCl}$ ). It was also observed that complete inhibition of $E$. histolytica adherence to target cells or colonic mucins is not observed, even when the lectin is blocked with high concentrations of galactose (100 to $500 \mathrm{mM})$ or GalNAc monomers [5], suggesting that other molecules than lectin could participate in the adhesion event. We have observed by Atomic Force Microscopy (AFM) the ameba surface and probed the interaction force between E. histolytica and chondroitin-6-sulphate (C6S). We have used several substrates to adhere trophozoites. The best reproducibility in sample preparation was obtained with fibronectin-coated coverslips and when the cells were fixed with paraformaldehyde. The images obtained with the AFM showed that the trophozoite exhibits an irregular surface. Pseudopods and waving adhesion plaques could be observed (fig.1). Force spectroscopy analysis showed that the trophozoite surface strongly interacts with C6Sfunctionalized tips (fig.2). During cantilever retraction, attractive force peaks were observed at distances up to $1.3 \mu \mathrm{m}$ above the trophozoite surface. Statistical analysis of the force distributions collected for five samples showed a reproducible $2.2 \mathrm{nN}$ mean adhesion force. We observed a reduction of the adhesion force and of the interaction distance after addition of galactose to the buffer solution suggesting that the observed interaction is also Gal/GalNAclectin-mediated (fig.3).

Supports: CNPq-PRONEX, Faperj, CAPES 
[1] Mann BJ, Torian BE, Vedvick TS, and Petri WAJr. Proc. Natl. Acad. Sci. U. S. A 88, (1991)

[2] Ravdin J I and Guerrant RL. J. Clin. Invest 68 (1981)

[3] Chadee K, Johnson ML, Orozco E, Petri WA Jr, and Ravdin JI. J. Infect. Dis. 158 (1988)

[4] Petri WA Jr. Crit Rev. Clin. Lab Sci. 33 (1996)

[5] Espinosa-Cantelano and Martinez-Palomo Curr Opin Infect Dis 13 (2000)

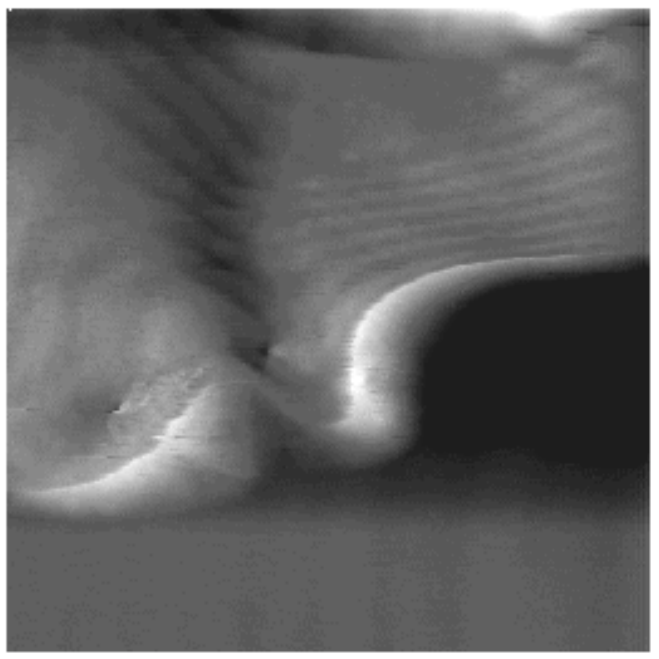

FIG 1

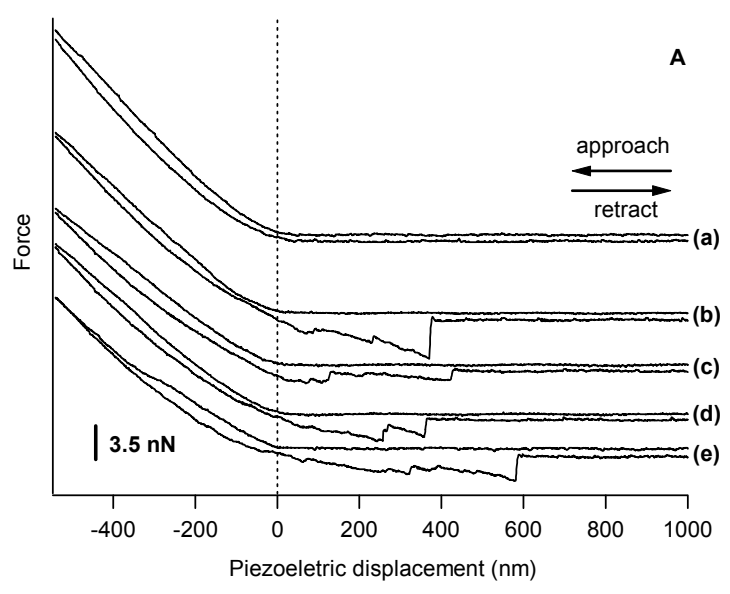

FIG 2

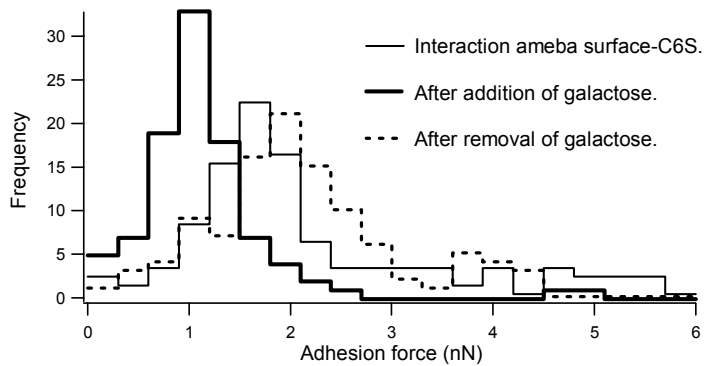

FIG 3 